\title{
Efficacy of low-dose valganciclovir in CMV R+ lung transplant recipients: a retrospective comparative analysis
}

\author{
Jessica Hunt, ${ }^{1}$ Kristina M. Chapple, ${ }^{2}$ Aasya Nasar, ${ }^{1}$ Lauren Cherrier, ${ }^{1}$ Rajat Walia ${ }^{3}$ \\ ${ }^{1}$ Department of Pharmacy; ${ }^{2}$ Department of Biostatistics and Neurosurgery; ${ }^{3}$ Department of Pulmonology, St. Joseph's \\ Hospital and Medical Center, Phoenix AZ, USA
}

Background: Cytomegalovirus (CMV) infection is extremely common after lung transplant and can be associated with significant morbidity and mortality. Current practice suggests the use of $900 \mathrm{mg}$ daily of valganciclovir for CMV prophylaxis, but there is no literature assessing whether $450 \mathrm{mg}$ daily of valganciclovir is sufficient in intermediate CMV risk lung transplant recipients. Therefore, we sought to assess the role of low-dose valganciclovir (LDV) versus high-dose valganciclovir (HDV) prophylaxis in intermediate-risk $(\mathrm{R}+)$ recipients.

Methods: We conducted a retrospective analysis on lung transplant recipients at the Norton Thoracic Institute in Phoenix, Arizona looking at intermediate-risk patients that received either valganciclovir $450 \mathrm{mg}$ per day (LDV) or $900 \mathrm{mg} /$ day (HDV). All patients were followed for 1 year post-transplant for incidence of CMV viremia. The primary outcome was the rate of CMV viremia as determined by a positive CMV polymerase chain reaction $([\mathrm{PCR}]>2.7 \mathrm{log}$ copies $/ \mathrm{mL}$ ). Secondary outcomes included rate of adverse events, acute cellular rejection, and mortality.

Results: The primary analysis included 103 patients (55 in the LDV group, 48 in the HDV group). In the LDV group, 9 patients $(16.4 \%)$ developed CMV viremia compared to $4(8.3 \%)$ in the HDV group ( $\mathrm{p}=0.221)$ with no difference observed in adverse event rates between groups.

Conclusion: There was no statistical difference between groups for the primary outcome. However, the effect size demonstrated in this analysis may be of clinical relevance and valganciclovir $450 \mathrm{mg}$ daily would not be recommended in intermediate risk lung transplant recipients at this time. To confirm our results, further prospective studies enrolling larger patient populations are necessary.

Key words: Cytomegalovirus; lung transplantation; valganciclovir.

Correspondence: Lauren Cherrier, PharmD, St. Joseph's Hospital and Medical Center, 350 W Thomas Rd, Phoenix, AZ 85013, USA. Tel. +1.602.406.3000 - Fax: +1.602.512.6647.

E- mail: lauren.cherrier@dignityhealth.org

Contributions: All authors made substantial contributions to the conception of the work; the acquisition, analysis, and interpretation of data; and the drafting and revision of the article. All authors approved the final version of the article for publication.

Conflict of interest: This study contains no off-label use of valganciclovir, and none of the authors has a financial relationship with a commercial entity that has an interest in the subject of the presented material or other conflicts of interest to disclose.

Funding: No funding was received for this study.

Availability of data and materials: Individuals interested in viewing the raw data analyzed in this manuscript are encouraged to contact the corresponding author.

Ethics approval and consent to participate: This study was approved by the Institutional Review Board at St. Joseph's Hospital and Medical Center in Phoenix, Arizona, USA.

Consent for publication: Not applicable. 


\section{Introduction}

Cytomegalovirus (CMV) is one of the most common infections after solid organ transplantation and is associated with high morbidity and mortality [1]. In addition to the detrimental effects of CMV tissue invasion, the infection also places transplant recipients at a higher risk of rejection. Valganciclovir is currently the standard of care for CMV prophylaxis and treatment [2]. While guidelines recommend $900 \mathrm{mg}$ daily for intermediate risk patients, they also mention the use $450 \mathrm{mg}$ daily, which has been studied in the kidney transplant population. The guidelines state that there is currently no data to support or contradict the use of $450 \mathrm{mg}$ daily dosing for intermediate risk lung transplant patients and the lung transplant studies referenced do not address varying doses of valganciclovir in this population [3]. The lung transplant specific studies primarily focused on comparing valganciclovir to a historical control (ganciclovir) or assessing the duration of prophylaxis with $900 \mathrm{mg}$ valganciclovir daily [4-8].

The majority of the data regarding CMV prophylaxis dosing in the lung transplant population are extrapolated from renal transplant studies [9-13]. However, this comparison may not be appropriate, as pulmonary tissue is inherently more susceptible to CMV infection. The reported incidence of CMV infection in lung transplant patients without prophylaxis has been reported to be as high as $54 \%$ to $92 \%$ (compared to less than $50 \%$ in kidney transplant patients) [14]; therefore, direct comparisons across transplanted organs may not be appropriate. Although some studies have shown benefit with low-dose strategies in low- and intermediate-risk patients, many of those same studies raise concern for higher rates of CMV viremia and resistance $[10,11,13,15]$.

In accordance with the renal transplant findings, and in an attempt to limit drug costs and adverse events such as leukopenia, our center used $450 \mathrm{mg}$ valganciclovir daily as prophylaxis in intermediate-risk patients beginning in 2010. In 2017, our center increased our prophylaxis dose to $900 \mathrm{mg}$ daily due to suspected higher rates of CMV viremia in this population. This study was conducted to review the change in protocol, to evaluate the most appropriate prophylactic regimen, and to determine whether valganciclovir $450 \mathrm{mg}$ daily can be used effectively in intermediate risk lung transplant patients.

\section{Methods}

This study was approved by the Institutional Review Board at St. Joseph's Hospital and Medical Center in Phoenix, Arizona, USA. We retrospectively assessed the difference between 2 groups of lung transplant recipients who received different valganciclovir CMV prophylaxis protocols after undergoing a lung transplant at the Norton Thoracic Institute (NTI) in Phoenix, Arizona, USA. Patients were assigned to regimens based on their risk of CMV as determined by their pre-transplant CMV serology. The focus of this analysis was on intermediate risk $(\mathrm{R}+)$ patients. Patients undergoing transplant between March 2016 and December 2016 were designated as the low dose valganciclovir (LDV) CMV prophylaxis group and received $450 \mathrm{mg}$ daily. In February 2017, NTI revised its institutional protocol to a high dose valganciclovir (HDV) regimen, where intermediate recipients received $450 \mathrm{mg}$ twice daily. This group was classified as patients that underwent transplant from March 2017 to December 2017 (Figure 1). Data from January and February of 2017 were excluded for simplicity to allow for appropriate time for education and implementation of the new protocol. In both protocols, valganciclovir began on day 4 posttransplant, and continued indefinitely. Baseline characteristics, efficacy, adherence, and adverse events were all collected retrospectively for both groups. The primary outcome was the rate of CMV viremia as determined by a positive CMV polymerase chain reaction ([PCR] $>2.7 \log$ copies $/ \mathrm{mL}$ ). Secondary outcome measures included leukopenia (white blood cell nadir and quarterly averages), renal function (peak serum creatinine, quarterly averages, and associated creatinine clearance), biopsy-confirmed rejection (Grade A1-A4), and mortality. CMV PCR was assessed week-

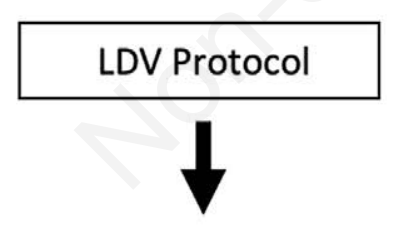

Transplanted from March 2016 to December 2016

\begin{tabular}{|c|}
\hline REGIMEN \\
Intermediate Risk (R+): \\
- Valganciclovir $450 \mathrm{mg}$ PO daily \\
*Cytomegalovirus immune globulin \\
postop day 0 and day 14
\end{tabular}

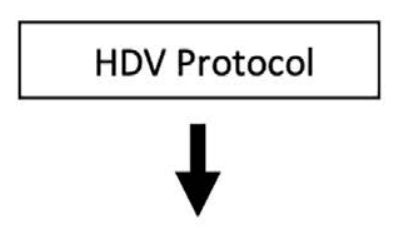

Transplanted from March 2017 to December 2017

\begin{tabular}{|c|}
\hline REGIMEN \\
Intermediate Risk (R+): \\
- Valganciclovir $450 \mathrm{mg}$ PO BID \\
(900 mg PO daily) \\
*No cytomegalovirus immunoglobulin \\
\hline
\end{tabular}


ly while patients were in the hospital and with routine labs posttransplant at months 1, 3, 6, and 12 .

Protocol adherence was monitored both inpatient and at outpatient clinic visits based on whether patients were receiving the correct dose of valganciclovir. However, due to the retrospective nature of this study, it was not possible to accurately assess patient compliance. Therefore protocol adherence was based solely on provider adherence to the specified protocol. Although there is no specific renal adjustment protocol for valganciclovir at our institution, patients with a regimen adjusted for renal function using standardized creatinine clearance cutoffs were considered adherent. Adjustments for hepatic dysfunction, or thrombocytopenia, however, were considered non-adherent to the protocol, as these adjustments were not considered standard for valganciclovir.

Means and standard deviation were used for continuous variable data. Percentages were used for categorical variables, including baseline characteristics. Chi-square and t-tests were used to assess primary and secondary outcomes. A p-value of $<0.05$ indicated statistical significance.

\section{Results}

A total of 103 intermediate-risk patients (55 in the LDV group and 48 in the HDV group) were included in the primary analysis. Patients were well matched for age, pre-transplant diagnosis, and lung allocation scores (Table 1). The majority of patients underwent bilateral lung transplant and received either basiliximab or the combination of rituximab and intravenous immunoglobulin for induction therapy. All patients were started on prednisone, mycophenolate mofetil, and tacrolimus for maintenance immunosuppression. Overall, no difference was seen in the rate of protocol adherence for either group in the first year posttransplant. In the LDV and HDV groups, patients were prescribed the appropriate per-protocol prophylaxis regimen more than $80 \%$ of the time
( $85.3 \%$ vs $81.29 \%$ respectively, $p=0.47)$. For the primary efficacy outcome, 9 of $55(16.4 \%)$ patients in the LDV group developed CMV viremia (positive CMV PCR); whereas 4 of the 48 patients in the HDV group developed CMV viremia $(8.3 \%$; $p=0.221$; Table 2 ). At the time of the primary outcome there were disparities between groups for protocol adherence. Of the HDV patients who developed a positive CMV PCR, only 2 out of 4 were adherent with the protocol regimen at the time of the positive PCR result. The other two patients had not started valganciclovir therapy: one patient had a positive PCR at day 3 posttransplant (prior to our standard initiation on day 4); the other patient had a positive PCR at day 11 posttransplant - this patient had valganciclovir withheld due to postoperative leukopenia. Given the few number of patients (only 50\%) in the intervention group on therapy at the time of the positive PCR, an exploratory outcome was conducted. In the per protocol analysis nine LDV patients $(16.4 \%$ ) had a positive PCR, compared to 2 patients $(4.16 \%)$ in the HDV group $(p=0.054)$.

There were no major differences in secondary outcomes for both groups. Average peak serum creatinine in the year following transplant were similar: $1.94 \mathrm{mg} / \mathrm{dL}$ in the LDV group vs 1.92 $\mathrm{mg} / \mathrm{dL}$ in the HDV group ( $\mathrm{p}=0.95$ ). The average worst creatinine clearance throughout the year was consequently well matched. Leukopenia was similar between groups, with an average nadir of 3.82 and 2.82 in the LDV and HDV groups, respectively $(p=0.211)$. Rejection and mortality rates remained consistent for both patient populations. Biopsy-confirmed rejection rates were similar at $38.2 \%(21 / 55)$ in the LDV and $41.6 \%(20 / 48)$ in the HDV groups $(\mathrm{p}=0.65)$. The rate of mortality was less than $10 \%$ in each group; LDV group: $7.3 \%$; HDV group: $8.3 \%(\mathrm{p}=0.84)$.

\section{Discussion}

Overall, this study showed a numerical reduction in the rate of CMV viremia after implementation of the new protocol. Though

Table 1. Baseline data for 103 patients at intermediate risk for developing CMV infection after lung transplant.

\begin{tabular}{|c|c|c|c|}
\hline Clinical characteristics & LDV ( $\mathrm{n}=55)$ & HDV (n=48) & $\mathbf{p}$ \\
\hline Mean age, years \pm SD & $59.22 \pm 13.3$ & $60.52 \pm 15.4$ & 0.65 \\
\hline Mean lung allocation score \pm SD & $43.7 \pm 15.1$ & $44.3 \pm 16.7$ & 0.84 \\
\hline Mean serum creatinine, $\mathrm{mg} / \mathrm{dl} \pm \mathrm{SD}$ & $0.68 \pm 0.2$ & $0.63 \pm 0.1$ & 0.12 \\
\hline Mean creatinine clearance, $\mathrm{ml} / \mathrm{min} \pm \mathrm{SD}$ & $92.89 \pm 36.5$ & $90.9 \pm 43.4$ & 0.80 \\
\hline $\begin{array}{l}\text { Transplant type } \\
\text { Unilateral, n (\%) } \\
\text { Bilateral, n (\%) } \\
\text { Retransplant, n (\%) }\end{array}$ & $\begin{array}{c}0(0.0) \\
55(100.0) \\
6(10.9)\end{array}$ & $\begin{array}{c}2(4.2) \\
45(93.8) \\
4(8.3)\end{array}$ & $\begin{array}{l}- \\
-\end{array}$ \\
\hline $\begin{array}{l}\text { Pre-transplant diagnosis, n (\%)* } \\
\text { Pulmonary fibrosis } \\
\text { Pulmonary hypertension } \\
\text { COPD } \\
\text { Cystic fibrosis } \\
\text { Rejection } \\
\text { Other }\end{array}$ & $\begin{array}{c}25(45.5) \\
5(9.1) \\
17(30.9) \\
4(7.3) \\
6(10.9) \\
3(5.5)\end{array}$ & $\begin{array}{l}24(50.0) \\
5(10.4) \\
12(25.0) \\
4(8.3) \\
4(8.3) \\
8(16.7)\end{array}$ & $\begin{array}{l}0.65 \\
0.82 \\
0.51 \\
0.84 \\
0.66 \\
0.07\end{array}$ \\
\hline $\begin{array}{l}\text { Induction agents, n (\%) } \\
\text { Rituximab/IVIG } \\
\text { Basiliximab } \\
\text { Thymoglobulin } \\
\text { Cytomegalovirus immune globulin use, n (\%) }\end{array}$ & $\begin{array}{c}21(38.2) \\
30(54.5) \\
4(7.3) \\
26(47.3)\end{array}$ & $\begin{array}{c}9(18.8) \\
38(79.2) \\
1(2.1) \\
3(6.3)\end{array}$ & $\begin{array}{c}0.029^{\circ} \\
-- \\
-- \\
- \\
<0.001\end{array}$ \\
\hline
\end{tabular}

LDV, low-dose valganciclovir; HDV, high-dose valganciclovir; ${ }^{*}$ some values total more than $100 \%$ due to overlap in disease categories; ${ }^{\circ}$ the overall $\chi^{2}$ was significant; however, based on standardized residuals there were no statistical differences for individual induction agents; COPD, chronic obstructive pulmonary disease; IVIG, intravenous immunoglobulin. 
the primary outcome was not statistically significant, the findings in this analysis may have clinical relevance. This study demonstrated a roughly $50 \%$ reduction in the rate of CMV viremia for patients receiving HDV.

A major strength of the present study was its closely matched baseline characteristics, which increases the internal validity of our patient population. In addition, our patient population has good external validity. In accordance with national trends, the most common pre-transplant diagnosis in our cohort was fibrotic lung disease, followed by chronic obstructive pulmonary disease [16]. In addition, the present study evaluated multiple parameters that may influence development of CMV viremia, including protocol adherence, immunosuppression, and medication adjustments in response to leukopenia, renal dysfunction, or rejection. Adherence with the prophylaxis regimen was previously reported. Discrepancies between groups were adjusted for with the assessment of the exploratory outcome, per-protocol CMV viremia. In addition, differences in the amount of immunosuppression between groups did not appear to be a confounding factor in the present study as all patients with a positive CMV PCR received the same maintenance immunosuppression with tacrolimus, mycophe- nolate mofetil, and prednisone. In those patients who developed CMV viremia, just 1 patient in each group had a tacrolimus level above the goal at the time of the positive PCR (levels were still less than $14 \mathrm{ng} / \mathrm{ml}$ ), so excessive immunosuppression does not appear to be a major confounder in this analysis. During the period of increased immunosuppression immediately posttransplant, the risk of infection is consequently higher $[2,14]$. As expected, this was demonstrated in our study, as CMV viremia was more common early after transplant. The average onset of CMV viremia was 32 days posttransplant (range: 3-113).

The present study has some limitations. Its retrospective nonrandomized design made it difficult to control for potential confounding variables. One potential confounder was the difference in the use of CMV immunoglobulin between groups. Our institution's new protocol also involved stopping the administration of CMV immunoglobulin. Within the LDV group, $47.3 \%$ of patients received cytomegalovirus immune globulin versus just $7 \%$ in the HDV group $(\mathrm{p} \leq 0.001)$. Based on the described mechanism of CMV immunoglobulin, it is reasonable to expect this discrepancy to influence reduced rates of CMV viremia in the LDV group; however, this was not the case. High-risk and low-risk patients at

Table 2. Outcomes of 103 patients at intermediate risk for CMV infection, based on prophylaxis protocol used.

\begin{tabular}{|c|c|c|c|}
\hline Variable* & LDV ( $\mathrm{n}=55)$ & HDV $(n=48)$ & p \\
\hline Percent adherence & $85.3 \pm 27.6$ & $81.3 \pm 28.3$ & 0.47 \\
\hline CMV viremia, n (\%) & $9(16.4)$ & $4(8.3)$ & 0.22 \\
\hline Following protocol at the time of positive PCR, $\mathrm{n}(\%)$ & $9 / 9(100.0)$ & $2 / 4(50.0)$ & - \\
\hline Per-protocol CMV viremia, n (\%) & $9(16.4)$ & $2(4.2)$ & 0.05 \\
\hline Biopsy-confirmed rejection, n (\%) & $21(38.2)$ & $20(41.7)$ & 0.65 \\
\hline Death, $n(\%)$ & $4(7.3)$ & $4(8.3)$ & 0.84 \\
\hline Peak serum creatinine, $\mathrm{mg} / \mathrm{dl}$ & $1.94 \pm 1.1$ & $1.92 \pm 1.3$ & 0.95 \\
\hline Lowest creatinine clearance, $\mathrm{ml} / \mathrm{min}$ & 45.4 & 38.3 & 0.29 \\
\hline Mean SCr Quarter 1, mg/dl & $1.0 \pm 0.6$ & $0.9 \pm 0.3$ & 0.29 \\
\hline Mean SCr Quarter 2, mg/dl & $1.2 \pm 0.3$ & $1.1 \pm 0.6$ & 0.46 \\
\hline Mean SCr Quarter 3, mg/dl & $1.8 \pm 3.8$ & $3.4 \pm 12.8$ & 0.47 \\
\hline Mean SCr Quarter 4, mg/dl & $1.9 \pm 4$ & $2.8 \pm 9.5$ & 0.59 \\
\hline Mean WBC nadir, cells/ıl & $3.4 \times 10^{3} \pm 2.4 \times 10^{3}$ & $2.8 \times 10^{3} \pm 2.17 \times 10^{3}$ & 0.21 \\
\hline Mean time to WBC nadir, days & $157 \pm 114.1$ & $165 \pm 115.4$ & 0.74 \\
\hline 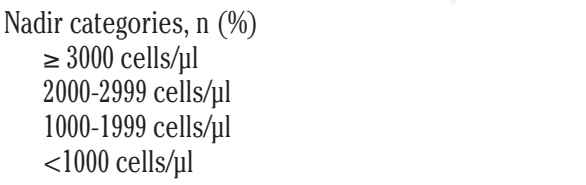 & $\begin{array}{c}18(32.7) \\
20(36.3) \\
13(23.6) \\
4(8.0)\end{array}$ & $\begin{array}{l}13(27.1) \\
16(33.3) \\
13(27.1) \\
6(12.5)\end{array}$ & $\begin{array}{c}0.76 \\
- \\
- \\
- \\
-\end{array}$ \\
\hline Mean WBC Quarter 1, cells/ $\mu l$ & $10.3 \times 10^{3} \pm 2.5 \times 10^{3}$ & $9.9 \times 10^{3} \pm 2.9 \times 10^{3}$ & - \\
\hline 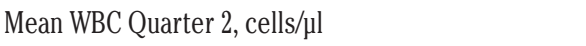 & $5.2 \times 10^{3} \pm 1.8 \times 10^{3}$ & $5.1 \times 10^{3} \pm 2.5 \times 10^{3}$ & - \\
\hline 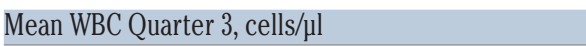 & $4.8 \times 10^{3} \pm 1.9 \times 10^{3}$ & $4.3 \times 10^{3} \pm 1.6 \times 10^{3}$ & - \\
\hline 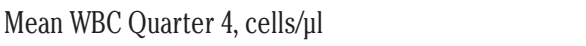 & $5.0 \times 10^{3} \pm 1.8 \times 10^{3}$ & $4.4 \times 10^{3} \pm 1.9 \times 10^{3}$ & - \\
\hline $\begin{array}{l}\text { Renal adjustments: Decreased, n (\%) } \\
\text { Valganciclovir } \\
\text { Sulfamethoxazole/trimethoprim } \\
\text { Immunosuppression goals }\end{array}$ & $\begin{array}{l}16(29.1) \\
28(50.9) \\
11(20.0)\end{array}$ & $\begin{array}{c}25(52.1) \\
18(37.5) \\
4(8.3)\end{array}$ & $\begin{array}{l}- \\
- \\
-\end{array}$ \\
\hline $\begin{array}{l}\text { Leukopenia adjustments } \\
\text { Decreased valganciclovir } \\
\text { Adjusted other medications }\end{array}$ & $\begin{array}{c}8(14.5) \\
19(34.5)\end{array}$ & $\begin{array}{l}12(25.0) \\
23(47.9)\end{array}$ & $\begin{array}{l}- \\
-\end{array}$ \\
\hline
\end{tabular}

*Values shown as mean \pm SD, unless indicated otherwise; LDV, low-dose valganciclovir; HDV, high-dose valganciclovir; CMV, cytomegalovirus; PCR, polymerase chain reaction; SCr, serum creatinine; WBC, white blood cells; ${ }^{\circ}$ adjustments included decreasing or holding mycophenolate mofetil; decreasing or holding sulfamethoxazole/trimethoprim, changing sulfamethoxazole/trimethoprim to atovaquone or pentamidine, or giving a granulocyte-colony stimulating factor. 
our institution (who did not undergo valganciclovir dose adjustments), demonstrated no change in rates of CMV viremia from one study period to the next, regardless of institutional adjustments to cytomegalovirus immunoglobulin. Previously published studies also support our findings, demonstrating a lack of benefit in terms of CMV infection rates after cytomegalovirus immunoglobulin administration [17-19]. This finding suggests that immunoglobulin use did not act as a major confounder in our analysis, as anticipated. Given the lack of demonstrated benefit in our study and in the literature, we currently recommend against its regular use for CMV prophylaxis in lung transplant recipients. Although our institution has general recommendations for adjusting valganciclovir for renal function, adjustments could not be entirely controlled for due to the retrospective nature of this analysis. Ultimately, adjustments occurred according to provider discretion and not a specified protocol. In the HDV group, valganciclovir appeared more likely to be reduced secondary to renal dysfunction than in the LDV group, in which sulfamethoxazole/trimethoprim, immunosuppression, and other nephrotoxic agents were typically adjusted first. On the other hand, there did not appear to be a major difference between groups in adjustments made secondary to leukopenia. The present study was not sufficiently powered to detect a difference in the primary outcome (current power calculation: $15 \%$ ). It is anticipated that 282 intermediate-risk patients would be required in each treatment group in order to achieve $80 \%$ power for the primary outcome. Our institution performs between 80 and 100 transplants per year; therefore, it would take roughly 3 years of patient enrollment in each group to achieve adequate power, according to the above calculation (4 years total, including followup). To date, the new protocol has been in effect for 2 years. Despite the power limitation, this study does have a much larger sample size compared to previously published literature in the lung transplant population.

Overall, this analysis showed a trend toward benefit with the high-dose valganciclovir regimen. This benefit was evidenced by fewer cases of CMV viremia with no increase in adverse events. Even though the literature in kidney transplant has shown utility in dosing valganciclovir $450 \mathrm{mg}$ daily for their intermediate risk population, we did not find the same outcome in the lung transplant population. This research provides further insight into prophylactic dosing in lung transplant patients (specifically those classified as intermediate risk based on pre transplant serology), with an effect size for the primary outcome that may be clinically relevant and that adds to the body of literature supporting higher doses of valganciclovir for these patients. Future analyses will target larger patient populations in a prospective trial design to correct for potential confounders in the present study (i.e., immunoglobulin use, medication adjustments, and compliance). Study outcomes will further assess high dose vs low dose efficacy in addition to optimum duration of therapy.

\section{Abbreviations}

CMV: cytomegalovirus;

HDV: high-dose valganciclovir;

LDV: low-dose valganciclovir;

PCR: polymerase chain reaction.

\section{References}

1. Avidan Y, Paul M, Rahamimov R, Bishara J, Samra Z, Edna S, et al. Selective low-dose valganciclovir for prevention of cytomegalovirus disease following kidney transplantation. J Infect 2008;57:236-40.

2. Kotton C, Kumar D, Caliendo A, Huprikar S, Chou S, Danziger-Isakov L, et al. The Third International Consensus Guidelines on the Management of cytomegalovirus in solidorgan transplantation. Transplantation 2018;102:900-31.

3. Razonable R, Humar A. Cytomegalovirus in solid organ transplantation. Am J Transplant 2013;13:s93-106.

4. Finlen Copeland C, Davis W, Snyder L, Banks M, Avery R, Davis R, et al. Long-term efficacy and safety of 12 months of valganciclovir prophylaxis compared with 3 months after lung transplantation: A single-center, long-term follow-up analysis from a randomized, controlled cytomegalovirus prevention trial. J Heart Lung Transpl 2011;30:990-6.

5. Humar A, Kumar D, Preiksaitis J, Boivin G, Siegal D, Fenton $\mathrm{J}$, et al. A trial of valganciclovir prophylaxis for cytomegalovirus prevention in lung transplant recipients. Am J Transplant 2005;5:1462-8.

6. Jaksch P, Zweytick B, Kerschner H, Hoda A, Keplinger M, Lang $\mathrm{G}$, et al. Cytomegalovirus prevention in high-risk lung transplant recipients: Comparison of 3- vs 12-month valganciclovir therapy. J Heart Lung Transplant 2009;28:670-5.

7. Palmer S, Limaye A, Banks M, Gallup D, Chapman J, Lawrence E, et al. Extended valganciclovir prophylaxis to prevent cytomegalovirus after lung transplantation. Ann of Intern Med 2010;152:761.

8. Zamora M, Nicolls M, Hodges T, Marquesen J, Astor T, Grazia $\mathrm{T}$, et al. Following universal prophylaxis with intravenous ganciclovir and cytomegalovirus immune globulin, valganciclovir is safe and effective for prevention of CMV infection following lung transplantation. Am J Transplant 2004;4:1635-42.

9. Chamberlain C, Penzak S, Alfaro R, Wesley R, Daniels C, Hale D, et al. Pharmacokinetics of low and maintenance dose valganciclovir in kidney transplant recipients. Am J Transplant 2008;8:1297-302.

10. Heldenbrand S, Li C, Cross R, DePiero K, Dick T, Ferguson K, et al. Multicenter evaluation of efficacy and safety of low-dose versus high-dose valganciclovir for prevention of cytomegalovirus disease in donor and recipient positive $(\mathrm{D}+/ \mathrm{R}+)$ renal transplant recipients. Transpl Infect Dis 2016;18:904-12.

11. Khan S, Sullivan T, Ali M, Dunn D, Patel G, Huprikar S. Lowdose valganciclovir for cytomegalovirus prophylaxis in intermediate-risk liver transplantation recipients. Liver Transpl 2018;24:616-2. A

12. Le Page A, Jager M, Kotton C, Simoons-Smit A, Rawlinson W. International survey of cytomegalovirus management in solid organ transplantation after the publication of consensus guidelines. Transplantation 2013;95:1455-60.

13. Stevens D, Sawinski D, Blumberg E, Galanakis N, Bloom R, Trofe-Clark J. Increased risk of breakthrough infection among cytomegalovirus donor-positive/recipient-negative kidney transplant recipients receiving lower-dose valganciclovir prophylaxis. Transpl Infect Dis 2015;17:163-73.

14. Azevedo L, Pierrotti L, Abdala E, Costa S, Strabelli T, Campos $\mathrm{S}$, et al. Cytomegalovirus infection in transplant recipients. Clinics 2015;70:515-23.

15. Eid A, Arthurs S, Deziel P, Wilhelm M, Razonable R. Emergence of drug-resistant cytomegalovirus in the era of valganciclovir prophylaxis: therapeutic implications and outcomes. Clin Transplant 2007;22:162-70.

16. Egan T, Edwards L. Effect of the lung allocation score on lung transplantation in the United States. J Heart Lung Transplant 
2016;35:433-9.

17. Kruger R, Paranjothi S, Storch G, Lynch J, Trulock E. Impact of prophylaxis with cytogam alone on the incidence of CMV viremia in CMV-seropositive lung transplant recipients. J Heart Lung Transplant 2003;22:754-63.

18. Rea F, Potena L, Yonan N, Wagner F, Calabrese F. Cytomegalovirus hyper immunoglobulin for CMV prophylaxis in thoracic transplantation. Transplantation 2016;100:S19-
26.

19. Aguado J, Gomez-Sanchez M, Lumbreras C, Delgado J, Lizasoain M, Otero J, et al. Prospective randomized trial of efficacy of ganciclovir versus that of anti-cytomegalovirus (CMV) immunoglobulin to prevent CMV disease in CMVseropositive heart transplant recipients treated with OKT3. Antimicrob Agents Chemother 1995;39:1643-5. 